\title{
Curing Black Hole Singularities with Local Scale Invariance
}

\author{
Predrag Dominis Prester \\ Department of Physics, University of Rijeka, Radmile Matejčić 2, 51000 Rijeka, Croatia \\ Correspondence should be addressed to Predrag Dominis Prester; pprester@phy.uniri.hr
}

Received 4 April 2016; Accepted 27 July 2016

Academic Editor: Xavier Leoncini

Copyright (C) 2016 Predrag Dominis Prester. This is an open access article distributed under the Creative Commons Attribution License, which permits unrestricted use, distribution, and reproduction in any medium, provided the original work is properly cited.

\begin{abstract}
We show that Weyl-invariant dilaton gravity provides a description of black holes without classical space-time singularities. Singularities appear due to the ill behaviour of gauge fixing conditions, one example being the gauge in which theory is classically equivalent to standard General Relativity. The main conclusions of our analysis are as follows: (1) singularities signal a phase transition from broken to unbroken phase of Weyl symmetry; (2) instead of a singularity, there is a "baby universe" or a white hole inside a black hole; (3) in the baby universe scenario, there is a critical mass after which reducing mass makes the black hole larger as viewed by outside observers; (4) if a black hole could be connected with white hole through the "singularity," this would require breakdown of (classical) geometric description; (5) the singularity of Schwarzschild BH solution is nongeneric and so it is dangerous to rely on it in deriving general results. Our results may have important consequences for resolving issues related to information loss puzzle. Though quantum effects are still crucial and may change the proposed classical picture, a position of building quantum theory around essentially regular classical solutions normally provides a much better starting point.
\end{abstract}

\section{Introduction}

Black hole (BH) solutions in General Relativity (GR) typically contain space-time singularities, that is, hypersurfaces which particles or observers may hit in finite proper time, but on which curvature blows up to infinity. A textbook example is Schwarzschild black hole:

$$
\begin{aligned}
d s^{2}= & -\left(1-\frac{2 G M}{r}\right) d t^{2}+\left(1-\frac{2 G M}{r}\right)^{-1} d r^{2} \\
& +r^{2}\left(d \theta^{2}+\sin ^{2} \theta d \varphi^{2}\right)
\end{aligned}
$$

where $G$ is Newton constant and $M$ is the mass of the $\mathrm{BH}$. A singularity is located at $r=0$, which can be established by calculating Kretschmann invariant $R_{\mu \nu \rho \sigma} R^{\mu \nu \rho \sigma} \sim r^{-6}$ and showing that all objects entering $\mathrm{BH}$ interior $(r<2 G M)$ end there in finite proper time. The general problem is that the classical physical description completely breaks down on such space-time singularities, and objects hitting them simply cease to exist (after being smashed by gravity). Unfortunately, there is a large body of theoretical analyses strongly indicating that singularities inside $\mathrm{BHs}$, as well as cosmological ones (Big
Bang being an example), are generically unavoidable in the classical GR.

As there is a neighbourhood around singularity where space-time curvature radii become smaller than Planck length, the usual philosophy is to seek rescue in the quantum gravity. However, standard physical reasoning would prefer a situation where classical singularities are not present, even if quantum description is important. Our goal here is to argue that one can achieve this in a rather conservative way without introducing new degrees of freedom, higher derivative, or nonlocal terms in the action ${ }^{1}$. In essence, one just needs to redefine the set of fundamental fields. For the reasons of simplicity and clarity, we will restrict our attention here to neutral spherically symmetric BHs, but we will argue that the main conclusions are valid more generally.

The outline of the paper is as follows. In Section 2, we set a stage with a brief review of Weyl-invariant dilaton gravity. In Section 3, we take one particular example of $\mathrm{BH}$ solution on which we explicitly demonstrate our main results. In Section 4, we argue that these results are valid for generic spherically symmetric BHs and possibly for all generic BHs which enclose a space-like singularity in GR description. We show that singularity of Schwarzschild $\mathrm{BH}$ solution is nongeneric 
and probably nonphysical. In Section 5, we conclude by summarising our main results and commenting on possible relevancy for information loss puzzle and cosmological singularities.

\section{Weyl-Invariant Dilaton Gravity}

Let us assume that the (physical) field content of a theory consists of the matter sector $\psi=\left\{\psi_{i}\right\}$ (all fields with nonzero spin except the graviton) and one scalar spin- 0 field $s$ and that the matter part of the action $\mathscr{A}_{\text {matt }}=\int d x^{4} \sqrt{-g} \mathscr{L}_{\text {matt }}(s, \psi)$ is locally scale invariant (Weyl-invariant). The Standard Model of particle physics with minimal Higgs sector is such a theory. Then, the simplest way to introduce dynamics to gravity by making the full classical action Weyl-invariant is to take the Lagrangian to be

$$
\begin{aligned}
\mathscr{L}_{\mathrm{WIDG}}= & \frac{1}{12}\left(\phi^{2}-s^{2}\right) R+\frac{1}{2}(\partial \phi)^{2}-\frac{1}{2}(\partial s)^{2} \\
& -V(\phi, s)+\mathscr{L}_{\text {matt }}(s, \psi),
\end{aligned}
$$

where $\phi$ is the new scalar field ("dilaton"). The scalar potential is restricted to the form

$$
V(\phi, s)=\phi^{4} f\left(\frac{s}{\phi}\right),
$$

where $f$ is an arbitrary function of the gauge-invariant $s / \phi$. The action then is invariant on Weyl transformations defined by

$$
\begin{gathered}
g^{\mu \nu}(x) \longrightarrow \Omega(x)^{2} g^{\mu \nu}(x), \\
\phi(x) \longrightarrow \Omega(x) \phi(x), \\
s(x) \longrightarrow \Omega(x) s(x), \\
\psi_{i}(x) \longrightarrow \Omega(x)^{d_{i}} \psi_{i}(x),
\end{gathered}
$$

where $\Omega(x) \neq 0$ is an otherwise arbitrary smooth function and $\left\{d_{i}\right\}$ are canonical scaling dimensions of the matter fields $\left\{\psi_{i}\right\}$. Such models are sometimes called Weyl-invariant dilaton gravity (WIDG) ${ }^{2}$. The formalism can be extended to include several scalar fields (e.g., describing nonminimal Higgs sector, inflaton, and axion) with possibly noncanonical kinetic terms [1] (and if one allows direct coupling of the dilaton $\phi$ to the matter sector, to any $\left.\mathscr{L}_{\text {matt }}\right)$. Though the general idea is rather old and goes back at least to [2], only recently did WIDG theory (2) receive serious attention from a phenomenological viewpoint, in particular in cosmological settings (see, e.g., [1, 3]) and in studies of a high-energy behaviour of the (canonical) quantum gravity $[4,5]$.

With Weyl invariance, an additional gauge symmetry is introduced. Though we have the additional field (dilaton $\phi(x)$ ), by using the gauge freedom (4), we can gauge-fix one field component so the total number of physical degrees of freedom is as in GR. In "normal" circumstances, $\phi$ and $g^{\mu \nu}$ are nonvanishing, thus causing Weyl symmetry to be spontaneously broken.
WIDG is not some exotic way of incorporating gravity into the theory. This is best seen in a so-called $E$-gauge which is defined by the following gauge-fixing condition:

$$
\left(\phi_{E}^{2}-s_{E}^{2}\right)=\text { const }=\frac{3}{4 \pi G_{N}} .
$$

This allows one to use the following parametrisation:

$$
\begin{gathered}
\phi_{E}(x)= \pm \sqrt{\frac{3}{4 \pi G_{N}}} \cosh \left(\sqrt{\frac{4 \pi G_{N}}{3}} \sigma(x)\right), \\
s_{E}(x)= \pm \sqrt{\frac{3}{4 \pi G_{N}}} \sinh \left(\sqrt{\frac{4 \pi G_{N}}{3}} \sigma(x)\right),
\end{gathered}
$$

so that in $E$-gauge WIDG Lagrangian becomes

$$
\mathscr{L}_{\mathrm{WIDG}}^{(E)}=\frac{R}{16 \pi G_{N}}-\frac{1}{2}(\partial \sigma)^{2}-V_{E}(\sigma)+\mathscr{L}_{\text {matt }}(\sigma, \psi),
$$

where $V_{E}(\sigma)=V\left(\phi_{E}, s_{E}\right)$. We see that in the domain where $E$-gauge is applicable WIDG is classically equivalent to the standard GR implementation of gravity, with $G$ playing the role of Newton constant. However, as $s / \phi$ is gauge-invariant, we see that $E$-gauge (5) (with finite $G$ ) can be defined only in the part of the configuration space satisfying $|\phi|>|s|$ with all configurations finitely separated from $|\phi|=|s|$ in $E$-gauge. One may say that WIDG is not an alternative theory to GR but its (Weyl-invariant) completion [1].

Our philosophy here is to take $\left\{\phi, s, g^{\mu \nu}, \psi\right\}$ as the fundamental fields. This means that, for physically acceptable solutions, these fields should be well behaved, at least in a class of gauges which we will call regular gauges. Note that the gauge-invariant scalar field $\sigma$, which from (6) is defined by

$$
\sigma=\sqrt{\frac{3}{4 \pi G_{N}}} \tanh ^{-1}\left(\frac{s}{\phi}\right),
$$

can be singular even when $\phi$ and $s$ are perfectly regular; this happens at the points where $|s / \phi| \rightarrow 1$. Recently, in a series of papers [6-8], it was demonstrated by explicit examples how cosmological singularities present in GR appear when $|s / \phi| \rightarrow 1$ and so are a consequence of a singular behaviour of $E$-gauge. Motivated by this result, we set as our goal here to investigate the nature of $\mathrm{BH}$ singularities in WIDG description.

\section{An Example}

Before discussing general $\mathrm{BHs}$, it is good to have some explicit examples for a demonstration. This section serves the purpose, and later on we will argue that the main results are generic in the WIDG description of BHs. Now, for practical purposes, we need an analytic solution, but with the nontrivial (i.e., not constant) scalar field because generic (dynamical) BH configurations are such. So, we can take one of the "hairy" spherically symmetric BH solutions from the literature, where the simplest contain just the metric and 
one physical scalar field as degrees of freedom. The simplest candidate appears to be BBM solution [9], but it was shown in [10] that this solution does not describe proper $\mathrm{BH}$ in the WIDG theory. The same applies to various generalisations of BBM solution, such as those described in [11-13 $]^{3}$.

One such simple analytic example of "hairy" $\mathrm{BH}$ solution is Zloshchastiev solution [14] $]^{4}$. In E-gauge, Lagrangian (7) is specified by $\mathscr{L}_{\text {matt }}=0$ and the scalar potential

$$
V_{E}(\sigma)=4 \lambda[3 \sinh \sigma-\sigma(\cosh \sigma+2)],
$$

where $\lambda$ is a coupling constant. The potential is obviously unbounded from below, which could make one uncomfortable. However, we will argue in Section 4 that this does not corrupt the analysis and that conclusions obtained below can be obtained from all known spherically symmetric proper $\mathrm{BH}$ solutions with nontrivial scalar field, also in physically acceptable theories, embedded in WIDG formalism.

This theory has two types of static spherically symmetric asymptotically flat $\mathrm{BH}$ solutions. Besides the standard Schwarzschild $\mathrm{BH}$ solution, for which space-time metric is as in (1) and $\sigma(x)=0$, there is also another "hairy" branch given by [14]

$$
\begin{aligned}
d s_{E}^{2} & =-N(r) d t^{2}+\frac{d r^{2}}{N(r)}+R(r)^{2}\left(d \theta^{2}+\sin ^{2} \theta d \varphi^{2}\right), \\
\sigma & =\ln \left(1+\frac{\kappa}{r}\right)
\end{aligned}
$$

where

$$
\begin{aligned}
& N(r)=1-\lambda\left[\kappa(2 r+\kappa)-2 R(r)^{2} \ln \left(1+\frac{\kappa}{r}\right)\right], \\
& R(r)=\sqrt{r(r+\kappa) .}
\end{aligned}
$$

The integration constant $\kappa$ is connected with ADM mass $M$ through $M=\lambda \kappa^{3} /\left(6 G_{N}\right)$. We have assumed $\lambda>0$ (there is $\{\sigma \rightarrow-\sigma, \lambda \rightarrow-\lambda\}$ symmetry), and we used the convention $16 \pi G_{N}=1$.

The main properties and the thermodynamical behaviour of this $\mathrm{BH}$ are qualitatively similar to those of Schwarzschild $\mathrm{BH}$. In $E$-gauge, there is a singularity at $r=0$, signalled by behaviour of curvature invariants $\left(R_{(E) \mu \nu \rho \sigma}\right)^{2},\left(R_{(E) \mu \nu}\right)^{2}$, and $R_{(E)}^{2}$ which all diverge as $\sim r^{-4}$ near $r=0$. The scalar field also diverges at the singularity as $\sigma \rightarrow-\ln r \rightarrow \infty$, which by (6) implies $\phi_{E} \rightarrow \infty$ and $s_{E} \rightarrow \infty$. For $\kappa>\lambda^{-1 / 2}$, there is a regular event horizon, located at $r=r_{h}>0$ with $r_{h}$ defined implicitly by $N\left(r_{h}\right)=0$, which hides singularity from the outer world in accord with Cosmic Censorship Conjecture. An object which enters $\mathrm{BH}$ interior $r<r_{h}$ unavoidably reaches $r=0$ in a finite proper time, so the space-time is geodesically incomplete. Larger $\mathrm{BH}$ (larger proper horizon area $\left.A_{(E) h}=4 \pi r_{h}\left(r_{h}+\kappa\right)\right)$ means larger entropy $S=$ $A_{(E) h} /\left(4 G_{N}\right)$ and larger mass $M$, but smaller $\mathrm{BH}$ temperature. Normal $\mathrm{BH}$ (with $\kappa>\lambda^{-1 / 2}$ ) by emitting Hawking radiation is expected to shrink (in the classical analysis) to $r_{h} \rightarrow 0$, $A_{(E) h} \rightarrow 0$ and to finite mass $M \rightarrow 1 /\left(6 G_{N} \lambda^{1 / 2}\right)$. When $\mathrm{BH}$ size becomes of the order of the Planck length $\left(r_{h} \sim 1\right)$, one expects quantum gravity to be strong and to dictate the final outcome of the process. In the rest of this section, we focus on the $\mathrm{BH}(10)-(11)$ and postpone the analysis of Schwarzschild $\mathrm{BH}$ to Section $4^{5}$.

We now proceed to show that, in WIDG picture, $r=$ 0 is not a space-time singularity but gauge artefact, by constructing gauges in which the solution is perfectly regular for $r>0$. First, we observe that $\sigma \rightarrow \infty$ for $r \rightarrow 0$, which when used in (8) gives $s / \phi \rightarrow 1$. This is a gauge-invariant result. Using this in (5) immediately signals that $E$-gauge breaks at $r=0$, so we need to find some nonsingular gauge. To keep manifest diff-covariance and also avoid possible higherderivative field transformations, let us restrict ourselves here to gauge-fixing conditions of the form $\mathscr{G}(\phi, s)=0$. We now show that gauges, which we call $p$-gauges, labeled by the parameter $p>1$ and defined by the condition

$$
\frac{1}{12}\left(\phi_{p}(x)^{2}-s_{p}(x)^{2}\right)=\exp (-2 p \sigma(x)), \quad p>1,
$$

do the job. The gauge-invariant scalar field $\sigma(x)$ is defined in (8). From (5) and (12), it follows that the transition from $E$-gauge to $p$-gauge is accomplished with the scaling factor $\Omega_{p}(x)=\exp (-p \sigma(x))$. Using this, we can immediately write solution (10)-(11) in the $p$-gauge, which is

$$
\begin{aligned}
& g_{(p) \mu \nu}=\left(1+\frac{\kappa}{r}\right)^{2 p} g_{(E) \mu \nu}, \\
& \left\{\begin{array}{l}
\phi_{p}(x) \\
s_{p}(x)
\end{array}\right\} \\
& =\sqrt{12}\left(1+\frac{\kappa}{r}\right)^{-p}\left\{\begin{array}{l}
\cosh \\
\sinh
\end{array}\right\}\left(\frac{1}{\sqrt{12}} \ln \left(1+\frac{\kappa}{r}\right)\right),
\end{aligned}
$$

where $g_{(E) \mu \nu}$ is given in (10)-(11).

(a) Regular Solution without Singularities for $r>0$. It is obvious that (13) describes asymptotically flat static spherically symmetric $\mathrm{BH}$ solution with event horizon located at the same $r_{h}$, obtained from $N\left(r_{h}\right)=0$. The main difference, compared to $E$-gauge result, is behaviour near $r=0$. Note first that, scalar fields $\phi_{p}$ and $s_{p}$ are well defined and vanishing in the limit $r \rightarrow 0$. Quadratic curvature invariants $R_{(p)}^{2}$, $\left(R_{(p) \mu \nu}\right)^{2}$, and $\left(R_{(p) \mu \nu \rho \sigma}\right)^{2}$ all behave as $\sim r^{4(p-1)}$ near $r=0$. In fact, it can be shown that any curvature invariant constructed by tensorial multiplication of $n$ Riemann tensors and $m$ covariant derivatives evaluated on metric (13) behaves like $\sim r^{(2 n+m)(p-1)}$ when $r \rightarrow 0$, so it is regular and vanishing for $p>1$. This already suggests that $r=0$ is not a singularity in $p$-gauges with $p>1$. To further understand properties of $r=0$ surface, it is necessary to analyse proper distances and particle trajectories near $r=0$. For $r \ll \kappa$, metric (13) is approximately given by

$$
\begin{gathered}
d s_{p}^{2} \approx\left(\frac{\kappa}{r}\right)^{2 p}\left[\left(\lambda \kappa^{2}-1\right) d t^{2}-\frac{d r^{2}}{\lambda \kappa^{2}-1}\right. \\
\left.+\kappa r\left(d \theta^{2}+\sin ^{2} \theta d \varphi^{2}\right)\right], \quad r \ll \kappa .
\end{gathered}
$$


We note that in the "static" (or Schwarzschild-like) coordinate system we employ (which is singular at the horizon), roles of $t$ and $r$ coordinates interchange for $r<r_{h}$, so $r$ variable becomes "time" coordinate and $t$ becomes spacial coordinate. From (14), we obtain that the proper time separation between two points with the same spatial coordinates $\left(r_{0}, t_{0}, \theta_{0}, \varphi_{0}\right)$ and $\left(r, t_{0}, \theta_{0}, \varphi_{0}\right)$ behaves for $r<r_{0} \ll \kappa$ as

$$
\Delta \tau_{p} \approx \frac{\kappa^{p}}{\sqrt{\lambda \kappa^{2}-1}}\left(r^{1-p}-r_{0}^{1-p}\right) .
$$

We see that timelike hypersurface $r=0$ is at an infinite proper distance from any point with fixed $r>0$. Similarly, for radial geodesics, the proper time also behaves as $\tau \sim r^{1-p}$.

However, classical trajectories of particles interacting with fields $\phi$ or $s$ are not described by geodesics, but by Weylinvariant action:

$$
\begin{aligned}
& \mathscr{A}_{\text {part }} \\
& \quad=-\int \phi(x) \chi\left(\frac{s(x)}{\phi(x)}\right) \sqrt{-g_{\mu \nu}(x) d x^{\mu} d x^{\nu}}+\cdots,
\end{aligned}
$$

where “..." stands for terms describing interaction with background fields from the matter sector which we neglect here. As for $r \ll \kappa$, we have $s / \phi \approx 1$; the simplest choice is to assume $\chi(s / \phi) \approx \chi_{0}=$ const. in (16) (though we will later entertain other possibilities). Then, one gets the following equations of motion for particle trajectories [15]:

$$
\begin{aligned}
\frac{d^{2} x^{\mu}}{d \lambda^{2}}+\Gamma_{\nu \rho}^{\mu} \frac{d x^{\nu}}{d \lambda} \frac{d x^{\rho}}{d \lambda} & \approx-\frac{1}{2} \partial^{\mu}\left(\phi^{2}\right), \\
\left(\frac{d \tau}{d \lambda}\right)^{2} & \approx \phi^{2}
\end{aligned}
$$

$$
\text { for } r \ll \kappa
$$

From (13), it follows that $\phi(r) \approx \sqrt{3}(r / \kappa)^{p-1 / \sqrt{12}}$ for $r \ll \kappa$. Using this and (14) in (17), we obtain that the proper time of particle radially approaching $r=0$ behaves as $\tau \sim r^{1-p}$, which means that $\tau \rightarrow \infty$ for $r \rightarrow 0$, so the worldlines of particles are not terminated at $r=0$ at finite proper time. Altogether, we obtain that the surface $r=0$ is located "at infinity" so that the space-time is in a sense complete.

(b) Importance of Quantum Gravity Effects. In p-gauges, it is still expected for quantum effects to be dominant near $r=0$. This can be seen by observing that effective Planck mass in $p$-gauge is given by

$$
\begin{aligned}
&\left(M_{\mathrm{Pl} \mathrm{eff}}\right)^{2}=\frac{1}{12}\left(\phi^{2}-s^{2}\right)=\left(1+\frac{\kappa}{r}\right)^{-2 p} \approx\left(\frac{r}{\kappa}\right)^{2 p}, \\
& r \ll \kappa .
\end{aligned}
$$

It becomes arbitrarily small near $r=0$, signalling the dominance of quantum gravity fluctuations in this region.

(c) Regularity and Observables. As observables are normally gauge-invariant objects, we must ascertain that there is a large enough set of diff- and Weyl-invariant quantities which are regular (i.e., finite when letting $r \rightarrow 0$ ). It is easy to see that there are an infinite number of nontrivial (i.e., not everywhere vanishing) regular local scalars including, for example,

$$
\begin{gathered}
\frac{s}{\phi}, \\
1-\frac{s^{2}}{\phi^{2}}, \\
\phi^{-4}\left(1-\frac{s^{2}}{\phi^{2}}\right)^{5} C_{\mu \nu \rho \sigma} C^{\mu \nu \rho \sigma},
\end{gathered}
$$

where $C_{\mu \nu \rho \sigma}$ is Weyl tensor, and all products of these scalars. In fact, one can take any Weyl-invariant scalar field and obtain from it (an infinite tower of) regular Weyl-invariant scalars by multiplying with $\left(1-s^{2} / \phi^{2}\right)^{k}$ with large enough exponent $k$ (and possibly other regular Weyl-invariant scalars $)^{6}$. In a similar fashion, we can also construct nonlocal global Weyl-invariant scalars using (single or multiple) integrals over space-time which are regular for $r \rightarrow 0$ (e.g., both kinetic and potential part of the WIDG action are such).

One important observable which must be addressed is the "physical proper time," which obviously is not the proper time defined from the metric due to its Weyl noninvariance. The solution can be found in (16), which must be taken as a definition of "the physical proper time." Now, if we take $\chi(x) \rightarrow$ const as $x \rightarrow 1$, then it is easy to show that the surface $r=0$ is separated by the finite physical time from the points with $r \neq 0$. In this case, it is possible to extend the solution, in the essentially smooth way $^{7}$, to the second asymptotic region of normal gravity or even to the antigravity regions of the parameter space in a similar manner as it was done in the cosmological setting in [6-8]. The second possibility happens when the function $\chi$ is of the form

$$
\chi\left(\frac{s}{\phi}\right)=\left(1-\frac{s^{2}}{\phi^{2}}\right)^{-k}
$$

For large enough $k$, physical time between $r=0$ and all $r \neq 0$ points becomes infinite. In this case, we can say that spacetime with $r>0$ is complete.

Which of the two possibilities is realised depends on the details of the physics near $r=0$. As argued above in (b), in the region $r \ll \kappa$, we expect full quantum gravity regime of some sort to be in operation so the proper analysis and behaviour of observables are impossible at the moment (though they are expected to be nonlocal; see, e.g., [16]). What we have shown is that, in the (semi)classical sense, there is no problem in constructing candidates for classical observables which are regular for $r \rightarrow 0$, which is at least a promising starting point toward the full quantum description.

(d) Baby Universe versus White Hole. The interior of the $\mathrm{BH}$ solution in $p$-gauges has a sort of baby universe type of metric. To see this, let us analyse two radial geodesics in angular 
coordinates $\theta$ and $\varphi$. The proper distance between geodesics as the function of time behaves as

$$
D(r) \propto r^{1 / 2-p}(r+\kappa)^{1 / 2+p} .
$$

The function $D(r)$ has a minimum at $r_{m}=(p-1 / 2) \kappa$ and tends to infinity when $r \rightarrow 0$ and $r \rightarrow \infty$. We see that radially infalling shell after the time $r_{m}$ (i.e., for $r<r_{m}$ ) expands its size. The baby universe is homogeneous but anisotropic. Now, taking into account not geometric but physical distances, this baby universe picture is unchanged in the second possibility stated in (c). In the first possibility, it is possible to pass $r=0$ and go into other asymptotic regions, which resembles the white hole scenario.

(e) E-Gauge Singularity as a Marker for the Phase Transition. In $p$-gauges, we have obtained that $\phi, s$, and $g^{\mu \nu}$ all vanish when $r \rightarrow 0$. It can be shown that the same applies to all fields with positive scaling dimension on Weyl rescaling, such as, for example, Weyl tensor $C^{\mu \nu}{ }_{\rho \sigma}^{8}$. As $\left(\phi, s, g^{\mu \nu}\right)=(0,0,0)$ is a fixed point of Weyl transformations, we see that, at a "singularity" $r=0$, there is a phase transition from broken to unbroken phase of Weyl symmetry.

(f) Thermodynamical Properties and the Fate of an Isolated $B H$. The $\mathrm{BH}$ entropy (as given by Wald formula), $\mathrm{BH}$ temperature (obtained from surface gravity which is conformally invariant), and ADM mass (defined by conformally invariant formula [17]) are invariant on Weyl transformations (4), so, in $p$-gauges, they are the same functions of parameters $\lambda$ and $\kappa$ as when calculated in $E$-gauge (i.e., in GR description). However, as the metric itself is not gauge-invariant, there are some new moments when one passes to regular gauges like $p$ gauges. Above, we have shown this by analysing proper time and distance inside $\mathrm{BH}$. Another example is the horizon area, which in $p$-gauge is different than in $E$-gauge, and is given by

$$
A_{(p) h}=\left(1+\frac{\kappa}{r_{h}}\right)^{2 p} A_{(E) h}=4 \pi r_{h}^{2}\left(1+\frac{\kappa}{r_{h}}\right)^{2 p+1} .
$$

For large BHs $\left(r_{h} \gg \ell_{\mathrm{Pl}}\right)$, we saw that $r_{h} \gg \kappa$ and $A_{(p) h} \approx$ $A_{(E) h}$. In fact, for large $\mathrm{BH}$ in $r>r_{h}$ region, the solution in $p$-gauge is approximately equal to solution in $E$-gauge, which is approximately equal to Schwarzschild solution. However, as black hole becomes smaller, for example, by Hawking radiation, and $r_{h}$ becomes of the order of $\kappa$, the factor $(1+$ $\kappa(r)^{2 p}$ becomes more and more important. A difference is dramatic for small black holes with $r_{h} \ll \kappa$ for which $A_{(p) h} \approx$ $\kappa^{2}(\kappa / r)^{2 p-1}$, which diverges in the limit $r_{h} \rightarrow 0$. In Figure 1 , we present plots for horizon area as a function of horizon radius, both in $p$-gauge (with $p=2$ ) and in $E$-gauge. The function $A_{(p) h}\left(r_{h}\right)$ has a global minimum at $r_{h m}=(p-1 / 2) \kappa$, and such "minimal" BHs are characterised by $\lambda \kappa_{m}^{2} \approx 3 p$. Again, in the baby universe scenario, the above analysis remains qualitatively the same after geometric proper distances are substituted with physical proper distances.

The properties of $\mathrm{BH}$ solution in $p$-gauges give a new twist to the ongoing debate on the question of what happens in the final phase of evaporation of $\mathrm{BHs}$, which is crucially

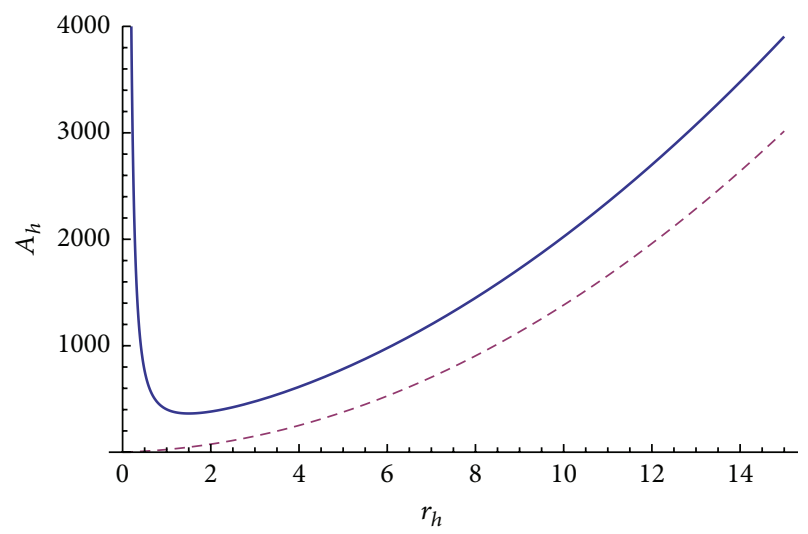

FIGURE 1: The figure shows horizon areas $A_{h}$ in $p$-gauge (bold line) and in $E$-gauge (dashed line) as a function of coordinate horizon radius $r_{h}$, for parameters $p=2$ and $\kappa=1$. The mass $M$ of the $\mathrm{BH}$ is gauge-independent, and $M / \kappa$ is a monotonic function of $r_{h} / \kappa$ (defined implicitly by $N\left(r_{h}\right)=0$ ). The exact profile of $A_{h}$ is gaugedependent, but the divergence as $r_{h} \rightarrow 0$ is a generic feature in all regular gauges.

important for discussions going around information loss paradox. As an isolated $\mathrm{BH}$, by emitting Hawking radiation, reaches the final phase of evaporation, the question is, does it evaporate completely or there remains some sort of a remnant (baby universe, Planck-size quantum remnant, etc.)? As the resolution depends on a yet not enough understood property of the quantum gravity, this is an open question. Yet, it is frequently claimed that the option with remnant is not valid or highly improbable. Standard arguments typically use one (or both) of the following properties of BH in GR: (1) "small internal volume" (consequence of the classical singularity) and (2) "small size" as viewed from the outside (a consequence that classically $A_{(E) h} \rightarrow 0$ as $r_{h} \rightarrow 0$ ). However, we have shown that, in regular gauges, like $p$-gauges, neither of these properties is present classically, first because there may be a baby universe (or a white hole) inside the $\mathrm{BH}$ and second because the horizon "explodes" in the final stage of evaporation (i.e., $A_{(p) h} \rightarrow \infty$ for $r_{h} \rightarrow 0$ ) in the case of the baby universe scenario. Of course, one still needs knowledge of the quantum gravity to fully answer the question of what finally happens with the black hole, but, in WIDG theories, it appears that the possibility of some sort of a $\mathrm{BH}$ remnant (or white hole) cannot be so easily dismissed.

(g) Generic Behaviour in Regular Gauges. We have seen that $|s(x) / \phi(x)| \rightarrow 1$ when $r \rightarrow 0$, so that $E$-gauge breaks when singularity is approached, and one needs to use another gauge which is regular in this limit. We have been able to construct a class of gauges, $p$-gauges (12), which are regular in the sense that all degrees of freedom $\left(\left\{\phi, s, g^{\mu \nu}\right\}\right)$ are regular everywhere. It can be shown that while details depend on the choice of the gauge, the generic features described in (a)(f) are gauge-independent in the sense that they are valid in all regular gauges. We used $p$-gauges just for demonstration purposes. Let us note that there may be other constraints on gauges to be regular, coming from the perturbation 
analysis (we discussed this in Section 4), quantisation, and/or asymptotic behaviour at $r \rightarrow 0$.

On the example of $\mathrm{BH}$ studied above, we can understand why the new properties discovered here in $p$-gauge (and valid in all regular gauges) were not found before. E-gauge (5) and $c$-gauge (defined by $\left.\phi(x)=\phi_{0}\right)$ do not cover $(\phi, s)=(0,0)$ point, which makes them inappropriate for studying $\mathrm{BH}$ solution near $r=0$, and this explains the presence of singularity in these gauges. It is easy to show that, in $s$-gauge (obtained by putting $p=1 / 2$ in (12)), the metric of this $\mathrm{BH}$ solution is also singular at $r=0$. The classical equations in the four mentioned gauges have the form of standard GR (minimally coupled fields), nonminimal GR with conformally coupled fields, and GR in "string-frame." These are the most studied formulations of gravity with 2-derivative actions in the context of constructing classical solutions, and as we see they all correspond to singular gauges.

\section{Generalisation}

One can check that the analysis from the previous section can be repeated, with essentially the same conclusions, for any of the known spherically symmetric BH solutions in GR with nontrivial scalar field, regardless of whether it is asymptotically flat or AdS, as long as they describe proper BHs from the viewpoint of the WIDG formulation of gravity. In particular, this is true for charged dilatonic $\mathrm{BH}$ solutions in low energy superstring effective theories and string inspired theories reviewed in $[18,19]$ or for $\mathrm{BHs}$ with non-Abelian hair reviewed in [20], which all have stable classical vacuum. The key property is that the scalar field in all these solutions is of the form $\sigma(r) \approx C_{1} \ln \left(r-r_{s}\right)+C_{2}$ near the singularity $r=r_{s}$ (in "Schwarzschild-like" coordinates), which then enables one to construct gauges, for example, $p$-gauges (12) with $p$ larger than some $p_{0}$, in which the solution is regular everywhere and analysis from the previous section can be readily performed.

For example, let us take as our second example the string inspired theory containing the scalar field $\sigma$ and $U(1)$ gauge field $A_{\mu}$, with the Lagrangian given by

$$
\mathscr{L}_{E}=R_{E}-\frac{1}{2}\left(\partial_{\mu} \sigma\right)^{2}-e^{-\sigma}\left(F_{\mu \nu}\right)^{2}
$$

where $F_{\mu \nu}=\partial_{\mu} A_{\nu}-\partial_{\nu} A_{\mu}$, and again using the convention $16 \pi G_{N}=1$. Contrary to the example used in the previous section, this theory does not have the problem with the classical stability of the vacuum. It was shown in [21] that there is a classical solution given by

$$
\begin{aligned}
d s_{E}^{2}= & -\left(1-\frac{2 M}{r}\right) d t^{2}+\left(1-\frac{2 M}{r}\right)^{-1} d r^{2} \\
& +r\left(r-\frac{Q^{2}}{M}\right) d \Omega_{2}, \\
\sigma(r)= & \ln \left(1-\frac{Q^{2}}{M r}\right), \\
F_{r t}= & \frac{Q}{r^{2}} .
\end{aligned}
$$

This solution describes electrically charged asymptotically flat $\mathrm{BH}$ where $M$ is the mass and $\mathrm{Q}$ is the electric charge $Q$. Event horizon is located at $r_{h}=2 M$ and singularity at $r_{s}=Q^{2} / M$, so the radial coordinate in $E$-gauge is defined only for $r>Q^{2} / M$. We can closely follow the procedure from the previous section, show that in $p$-gauges (12), with $p>1$, solution is regular in the sense described there, and obtain the same general conclusions as in (a)-(g).

However, our construction obviously does not work for $\mathrm{BH}$ solutions in which scalar field is trivial, that is, constant in the whole space-time, which include the very important case of Schwarzschild BHs. Moreover, for a large class of scalar potentials $V_{E}(\sigma)$, there are "no-hair" theorems guarantying that Schwarzschild $\mathrm{BH}$ with constant scalar field is the unique spherically symmetric $\mathrm{BH}$ solution regular everywhere except at a central singularity ${ }^{9}$. It is an obvious consequence of (12) that in this case solution in $p$-gauge has the same form as in $E$-gauge so it is singular at $r=0$. Constant $\sigma$ means, by (6), that both "fundamental" scalar fields $\phi_{E}$ and $s_{E}$ are constant and moreover that $|s(x)|<|\phi(x)|$ everywhere including the singularity $r=0$. We see that Schwarzschild $\mathrm{BH}$ violates the central conjecture that GR singularities are artefacts of invalidity of $E$-gauge when $|s|=|\phi|$. Does this kill our argument? No. The clue lies in understanding that Schwarzschild $\mathrm{BH}$ is a singular solution in the space of all $\mathrm{BH}$ solutions which is realised only for idealised initial conditions (of the zero measure). When one considers perturbations around it, either coming from matter/fields infalling or sitting outside $\mathrm{BH}[22]$, or when $\mathrm{BH}$ is inside the dynamical background (like FRW metric of cosmological setting [23]), the perturbed fields generally behave near the singularity $r=0$ as $\Phi(x) \sim$ $\ln (r)$ for scalar fields and as $\Phi(x) \sim r^{-n}$ for higher-spin fields (in the "Schwarzschild-like" coordinates). We see that, for generic solutions, corresponding to generic initial conditions, we have $\sigma \sim \ln (r) \rightarrow \infty$ which directly implies $|s / \phi| \rightarrow 1$ when $r \rightarrow 0$. Let us mention that general nonperturbative analysis of space-like singularities confirms that logarithmic behaviour of scalar fields is generic [24]. We then conjecture that there are regular gauges in which generic $\mathrm{BH}$ solutions are described by finite and regular $\phi(x), s(x)$, and $g_{\mu \nu}(x)$ (and other matter fields) in the whole space-time.

The full procedure of finding well-defined gauges in which solutions are regular cannot be performed simply because there are no analytic solutions for such "perturbed" Schwarzschild BHs. To get a taste of what is happening, let us consider a related problem of regulating generic spherically symmetric homogeneous solutions near a space-like singularity in GR. In GR (E-gauge) with a massless scalar with vanishing potential $V_{E}(\sigma)=0$, such generic solutions near space-time singularity, located at $r=0$, are approximately of the form [25]

$$
\begin{aligned}
d s_{E}^{2} & \approx r^{\beta^{2}-1} d t^{2}-A r^{\beta^{2}+1} d r^{2}+r^{2}\left(d \theta^{2}+\sin ^{2} \theta d \varphi^{2}\right), \\
\sigma & \approx \beta \ln r+C,
\end{aligned}
$$

where $C, \beta$, and $A>0$ are independent constants of integration and $r=0$ defines space-time singularity. The singularity of Schwarzschild $\mathrm{BH}$ with mass $M$ is obtained by setting $\beta=0$ 


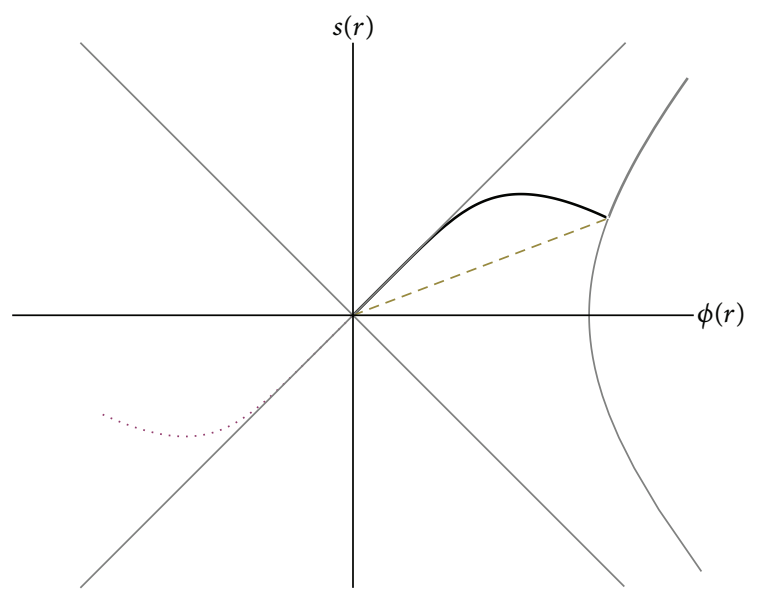

FIGURE 2: The figure shows a nongeneric nature of Schwarzschild singularity and also a singular nature of $E$-gauge (GR description) by plotting solutions in $(\phi, s)$-plane. The black thick line represents a $\mathrm{BH}$ solution with generic space-like ( $E$-gauge) singularity which is in regular gauges characterised by $(\phi, s) \rightarrow(0,0)$ and $|s / \phi| \rightarrow$ 1 (independently of values of scalar fields in asymptotic infinity). The dashed line represents Schwarzschild $\mathrm{BH}$ which is in all gauges characterised by $|s / \phi|=$ const $<1$. The point in which two solutions coincide corresponds to asymptotic infinity $r \rightarrow \infty$. Solutions in $E$ gauge are obtained by projecting on a hyperbola (thin gray line). We see that, for generic solution, this projection (thick gray line) is illdefined when $(\phi, s) \rightarrow(0,0)$ in regular gauge, and this manifests itself as space-time singularity in $E$-gauge. The dotted thick line represents a white hole extension (in region with negative $\phi$ and $s$ ). Thin grey diagonal lines are straight lines $|\phi|=|s|$.

and $A=1 /(2 G M)$. We explicitly see the exceptional character of Schwarzschild BH singularity ${ }^{10}$. For all other solutions, one has $\beta \neq 0$ and logarithmic behaviour of the field $\sigma$. It is easy to find gauges in which solutions with $\beta \neq 0$ are regular, for example, by choosing $\Omega=\exp \left(-\sigma^{2}\right)$ as a Weyl-rescaling factor doing transition from $E$-gauge ${ }^{11}$. In all such gauges, the same picture emerges as in the example studied in Section 3, again confirming generic nature of our results.

There are gauges in which Schwarzschild $\mathrm{BH}$ is regular, obtained by using higher-derivative terms. An example, with the lowest order in derivatives, is a class of gauges obtained by applying Weyl-factor

$$
\Omega=\left(1+c\left(R_{\mu \nu \rho \sigma}\right)^{4}\right)^{-p}, \quad c>0, p>\frac{1}{8},
$$

on the $E$-gauge fields ${ }^{12}$. However, in these gauges, Schwarzschild solution is still exceptional because it has $|s / \phi|=$ const $<1$ (this constant is determined by the asymptotic value at $r \rightarrow \infty$ ) while generic solutions behave as $|s / \phi| \rightarrow 1$ in $r \rightarrow 0$ limit. This is pictorially described in Figure 2. Besides, in such gauges, one introduces higher-derivative terms which are usually unwanted (though they should not be dismissed a priori).

The emerging picture from our analysis is that Schwarzschild $\mathrm{BH}$ is a singular solution also in WIDG formulation and should not be considered "microscopically" relevant (it can be used only as an average over ensemble of regular classical solutions). This means that it may be misleading to use calculations based only on Schwarzschild $\mathrm{BH}$ as a guideline in circumstances where microscopic properties of the near-singularity region play an important role. This is not so surprising as the same is probably true for other eternal $\mathrm{BH}$ solutions, like Reissner-Nordström or Kerr BHs, which poses inner Cauchy horizons which appear to be unstable under small perturbations. The morale is, to understand what is happening inside the $\mathrm{BHs}$, one must consider solutions with initial conditions which are generic enough.

\section{Conclusion}

We have demonstrated that a two-derivative Weyl-invariant formulation of gravity provides a description of black holes essentially without classical singularities. Appearance of singularities in standard formulation of gravity (GR) is simply an artefact of the fact that particular choice of the gauge fixing of local scale symmetry, in which the theory is equivalent to GR, is not well defined at singularities. By studying classical black hole solutions in regular gauges, in which fundamental fields are all well defined in the whole space-time, we obtained the following main results: (1) singularity is a signal of a phase transition from broken to unbroken phase of Weyl symmetry; (2) instead of a singularity, there is a "baby universe" or white hole inside a black hole; (3) as black hole decreases its mass (e.g., by Hawking radiation), there is a critical mass after which reducing mass makes the black hole bigger as viewed by outside observers (in the baby universe scenario). We emphasise that these results are obtained in the theory which is classically completion of GR, without introducing new physical fields (normal or phantom) or higher-derivative terms in the action. In fact, our classical analysis is essentially equivalent to using particular field redefinitions, but allowing the possible natural extension of the parameter space (important in white hole scenario). The essential requirement is that there is at least one physical scalar field in the theory with the properties which Standard Model Higgs field fulfils.

These results may be of relevance for issues related to unitarity of the evolution of black holes, indicating that $\mathrm{BHs}$ do not evaporate completely through the process of Hawking radiation and that some information, of possibly unlimited amount, may simply go into remnant baby universe or white hole. Such scenarios may offer a resolution (see, e.g., [26, 27], for a review) of Hawking's information loss paradox without resorting to exotic concepts, such as "firewalls," breaking of semiclassical approximation, or violation of unitarity.

It is interesting to compare our findings with those obtained in the context of cosmological singularities inside the same framework of Weyl-invariant formulation of gravity and presented in $[7,8]$. The common observation is that generic solutions in regular gauges behave as $(\phi, s) \rightarrow(0,0)$ and $|s / \phi| \rightarrow 1$ as one approaches surfaces which are homogeneous space-like singularities in $E$-gauge, while the simple solutions, like highly symmetric FRW solutions (homogeneous and isotropic) and Schwarzschild $\mathrm{BH}$ (constant scalar fields), have different nongeneric behaviour. Also, any attempt to extend solutions through the GR singularity necessarily implies breakdown of classical space-time metric 
description, but there are an enough number of classical observables which are regular across the singularity which can be used to formally make such extensions ${ }^{13}$. These agreements indicate that our main conclusions may possibly generalise to all space-like singularities.

The analysis we presented is completely classical, and for several reasons it cannot be considered complete without mentioning quantum effects. First, one could worry about health of the Weyl invariance we used extensively, in view of the generally anomalous nature of scale symmetry. However, in theories with dilaton field, there are regularisation cases in which classical Weyl invariance is preserved in an effective action $[28,29]$. Second, indeed a very important reason is that the proposed classical resolution of singularities happens deeply in the quantum regime, so knowledge of the quantum gravity is necessary to know whether we can trust our classical picture of regular space-time (or picture with extension to white hole) or not. Needless to say, such knowledge is currently beyond our grasp. But building the quantum theory around regular classical solutions (situation in Weylinvariant formulation of gravity), as opposed to singular solutions (a situation in standard General Relativity), would normally be considered as a much better starting point.

\section{Competing Interests}

The author declares that there are no competing interests regarding the publication of this paper.

\section{Acknowledgments}

The author thanks L. Bonora, M. Cvitan, S. Pallua, and I. Smolic for stimulating discussions. The research was supported by the University of Rijeka under Research Support no. 13.12.1.4.05.

\section{Endnotes}

1. That some higher-derivative theories may resolve singularities is observed before (e.g., see [30-32]). However, such theories either include new degrees of freedom, have problems with unitarity, or are nonlocal.

2. In principle, one could add higher-curvature terms in the action; for example, at four derivatives, one allowed term is $\Delta \mathscr{L}_{C}=h(s / \phi) C^{2}$, where $C_{\mu \nu \rho \sigma}$ is the Weyl tensor and $h$ is an arbitrary function. As we want to stick to twoderivative theories, we will not include such terms here.

3. As shown in [10], from the viewpoint of WIDG theory, these solutions describe "collections" of space-time without an event horizon, such as wormholes and naked singularities.

4. We could instead use any of the proper "hairy" $\mathrm{BH}$ solutions from $[33,34]$. The results would be essentially the same.

5. In [14], it is argued that the above model, or its generalisation which includes cosmological constant, may be interesting from the cosmological perspective. However, we emphasise again that the sole purpose of this $\mathrm{BH}$ solution here is to provide us with an analytic nontrivial toy model which we use to build general arguments. Those who want more details about Zloshchastiev BH solution, properties of potential (9), and its possible relevance in cosmology should consult [14].

6. Of course, not all scalars are expected to be regular on all physical configurations. For example, in General Relativity with minimally coupled scalar field $\phi$ (especially if a global minimum of the potential is at $\phi=0$ ), such "singular" scalars are $\phi^{-k}, \phi^{-k} R$, and so forth, with $k>0$. We emphasise this trivial fact because of an objection, originally used in [35] in a cosmological context, that a Weyl-invariant $\left(\phi^{2}-s^{2}\right)^{-2} C^{2}$ is singular when $r \rightarrow 0$. However, it is not clear why this invariant should have such physical significance in WIDG gravity. On the contrary, from its form, it is quite possible that it belongs to the class of singular invariants which have the problem of dividing by zero.

7. Regular Weyl invariants behave smoothly over $r=0$.

8. This is valid for all definitions of time coordinate $\bar{t}=\bar{t}(r)$ in which surface $r=0$ is located at finite $\bar{t}_{0}=\bar{t}(0)$. For other choices of time variable, one gets that pullback of tensors on the surface $r=0$ is vanishing.

9. In the example from the previous section, potential (9) is unbounded from below, which is the reason why "nohair" theorems are avoided.

10. We also see that the BH solution (10)-(11), which we used as an example to demonstrate our claims, has a singularity which is of generic type.

11. The $p$-gauges we constructed in Section 3 are not completely satisfactory here because for fixed finite $p$ they would regulate only solutions (25) having $\beta \geq \beta_{p}$, where $\beta_{p}>0$ is a function of $p$.

12. In these gauges, classical action contains higher-derivative terms typically leading to new degrees of freedom and/or breaking of unitarity. For this reason, results obtained in such gauges should be treated with care.

13. After the first version of this paper was submitted, a paper [35] which emphasised singular behaviour of extensions through cosmological singularities was constructed in $[7,8]$. However, as explained in point (c) in Section 3 (see in particular endnote 3 ), we think that the particular argument used in [35] is too simplistic.

\section{References}

[1] I. Bars, P. Steinhardt, and N. Turok, "Local conformal symmetry in physics and cosmology," Physical Review D, vol. 89, no. 4, Article ID 043515, 15 pages, 2014.

[2] P. A. M. Dirac, "Long range forces and broken symmetries," Proceedings of the Royal Society of London A, vol. 333, p. 403, 1973.

[3] R. Kallosh and A. Linde, "Universality class in conformal inflation," Journal of Cosmology and Astroparticle Physics, vol. 1307, p. 002, 2013. 
[4] G. 't Hooft, "Probing the small distance structure of canonical quantum gravity using the conformal group," https://arxiv.org/ abs/1009.0669.

[5] G. 't Hooft, "A class of elementary particle models without any adjustable real parameters," Foundations of Physics, vol. 41, no. 12, pp. 1829-1856, 2011.

[6] I. Bars, S.-H. Chen, and N. Turok, "Geodesically complete analytic solutions for a cyclic universe," Physical Review D, vol. 84, no. 8, Article ID 083513, 2011.

[7] I. Bars, S.-H. Chen, P. J. Steinhardt, and N. Turok, "Antigravity and the big crunch/big bang transition," Physics Letters B, vol. 715, no. 1-3, pp. 278-281, 2012.

[8] I. Bars, S.-H. Chen, P. J. Steinhardt, and N. Turok, "Complete set of homogeneous isotropic analytic solutions in scalar-tensor cosmology with radiation and curvature," Physical Review D, vol. 86, no. 8, Article ID 083542, 20 pages, 2012.

[9] N. Bocharova, K. Bronnikov, and V. Melnikov, "An exact solution of the system of Einstein equations and mass-free scalar field," Vestnik Moskovskogo Universiteta, Seriya 3: Fizika, Astronomiya, vol. 25, no. 6, p. 80, 1970.

[10] P. Dominis Prester, "Field redefinitions, Weyl invariance and the nature of mavericks," Classical and Quantum Gravity, vol. 31, no. 15, Article ID 155006, 14 pages, 2014.

[11] J. D. Bekenstein, "Exact solutions of Einstein-conformal scalar equations," Annals of Physics, vol. 82, pp. 535-547, 1974.

[12] C. Martinez, R. Troncoso, and J. Zanelli, "De Sitter black hole with a conformally coupled scalar field in four dimensions," Physical Review D, vol. 67, no. 2, Article ID 024008, 3 pages, 2003.

[13] H. Cebeci and T. Dereli, "Conformal black hole solutions of axi dilaton gravity in D-dimensions," Physical Review D, vol. 65, Article ID 047501, 2002.

[14] K. G. Zloshchastiev, "On co-existence of black holes and scalar field," Physical Review Letters, vol. 94, Article ID 121101, 2005.

[15] J. D. Bekenstein, "Black holes with scalar charge," Annals of Physics, vol. 91, no. 1, pp. 75-82, 1975.

[16] S. B. Giddings, D. Marolf, and J. B. Hartle, "Observables in effective gravity," Physical Review D, vol. 74, no. 6, Article ID 064018, 2006.

[17] K. C. K. Chan, J. D. E. Creighton, and R. B. Mann, "Conserved masses in GHS Einstein and string black holes and consistent thermodynamics," Physical Review D, vol. 54, no. 6, pp. 38923899, 1996.

[18] G. T. Horowitz, "The dark side of string theory: black holes and black strings," https://arxiv.org/abs/hep-th/9210119.

[19] D. Youm, "Black holes and solitons in string theory," Physics Reports, vol. 316, no. 1-3, pp. 1-232, 1999.

[20] G. V. Lavrelashvili, "NonAbelian surprises in gravity," http:// arxiv.org/abs/gr-qc/9701049.

[21] G. W. Gibbons, "Antigravitating black hole solitons with scalar hair in $N=4$ supergravity," Nuclear Physics B, vol. 207, no. 2, pp. 337-349, 1982.

[22] A. G. Doroshkevich and I. D. Novikov, "Space-time and physical fields in black holes," Journal of Experimental and Theoretical Physics, vol. 74, p. 3, 1978.

[23] S. Chadburn and R. Gregory, "Time dependent black holes and scalar hair," Classical and Quantum Gravity, vol. 31, no. 19, Article ID 195006, 2014.

[24] V. A. Belinskii and I. M. Khalatnikov, "Effect of scalar and vector fields on the nature of the cosmological singularity," Zhurnal
Eksperimental'noi i Teoreticheskoi Fiziki, vol. 63, p. 1121, 1972, Soviet Physics Journal of Experimental and Theoretical Physics, vol. 36, pp. 591, 1973.

[25] L. M. Burko, "Homogeneous spacelike singularities inside spherical black holes," http://arxiv.org/abs/gr-qc/9711012.

[26] S. D. H. Hsu, "Spacetime topology change and black hole information," Physics Letters B, vol. 644, no. 1, pp. 67-71, 2007.

[27] S. Hossenfelder and L. Smolin, "Conservative solutions to the black hole information problem," Physical Review D, vol. 81, no. 6, Article ID 064009, 13 pages, 2010.

[28] R. Percacci, "Renormalization group flow of Weyl invariant dilaton gravity," New Journal of Physics, vol. 13, Article ID 125013, 20 pages, 2011.

[29] A. Codello, G. D’Odorico, C. Pagani, and R. Percacci, "The renormalization group and Weyl invariance," Classical and Quantum Gravity, vol. 30, no. 11, 115015, 22 pages, 2013.

[30] P. Kanti, J. Rizos, and K. Tamvakis, "Singularity-free cosmological solutions in quadratic gravity," Physical Review D, vol. 59, no. 8, Article ID 083512, 1999.

[31] L. Modesto, "Super-renormalizable Quantum Gravity," Physical Review D, vol. 86, no. 4, Article ID 044005, 2012.

[32] T. Biswas, E. Gerwick, T. Koivisto, and A. Mazumdar, "Towards singularity- and ghost-free theories of gravity," Physical Review Letters, vol. 108, no. 3, Article ID 031101, 4 pages, 2012.

[33] A. Anabalon, D. Astefanesei, and R. Mann, "Exact asymptotically flat charged hairy black holes with a dilaton potential," Journal of High Energy Physics, vol. 2013, p. 184, 2013.

[34] A. Anabalon, "Exact Hairy Black Holes," https://arxiv.org/abs/ 1211.2765.

[35] J. J. M. Carrasco, W. Chemissany, and R. Kallosh, "Journeys through antigravity?" Journal of High Energy Physics, vol. 2014, no. 1, article 130, 2014. 


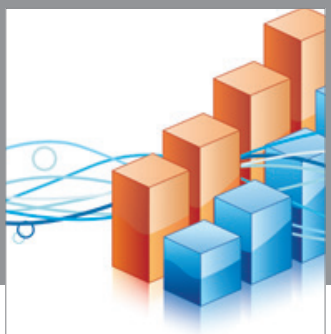

Advances in

Operations Research

vatem alat4

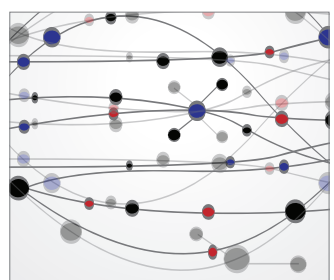

\section{The Scientific} World Journal
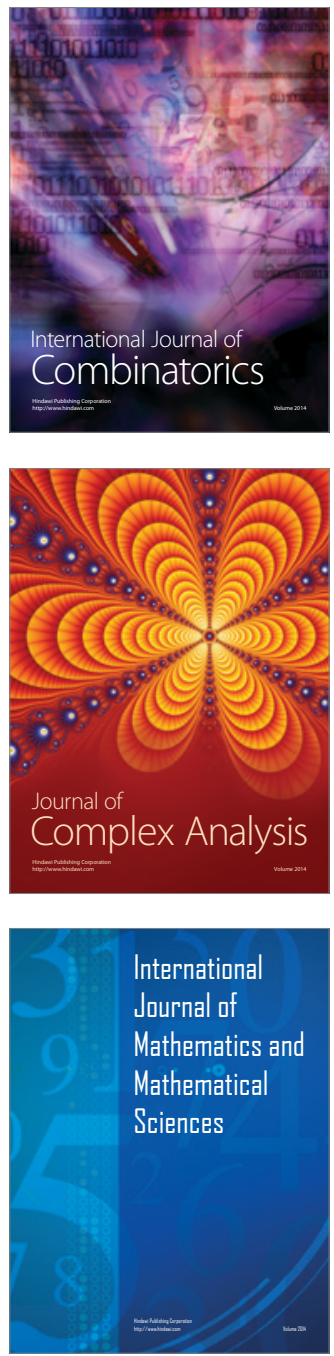
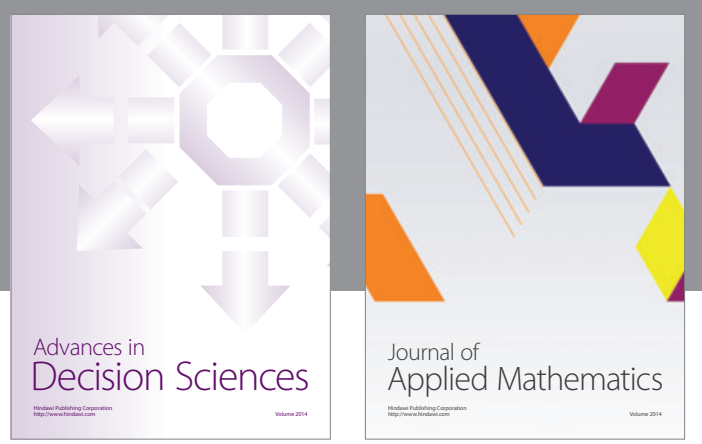

Algebra

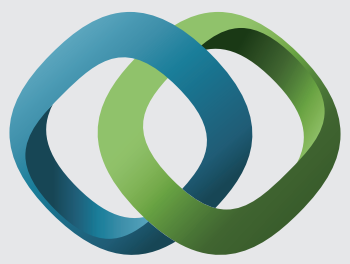

\section{Hindawi}

Submit your manuscripts at

http://www.hindawi.com
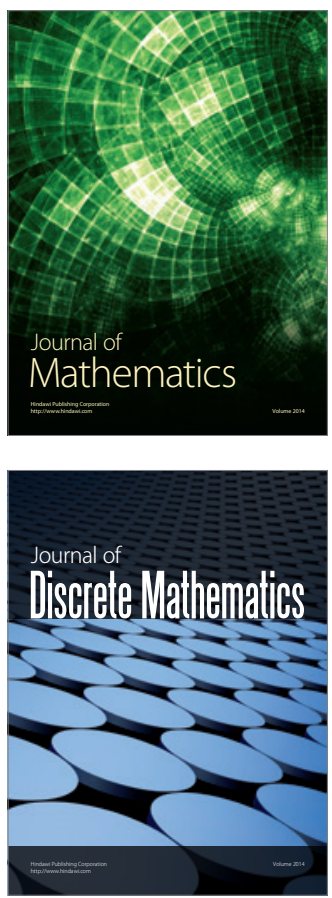

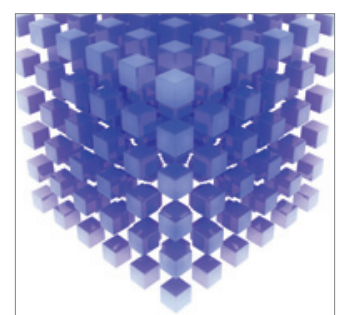

Mathematical Problems in Engineering
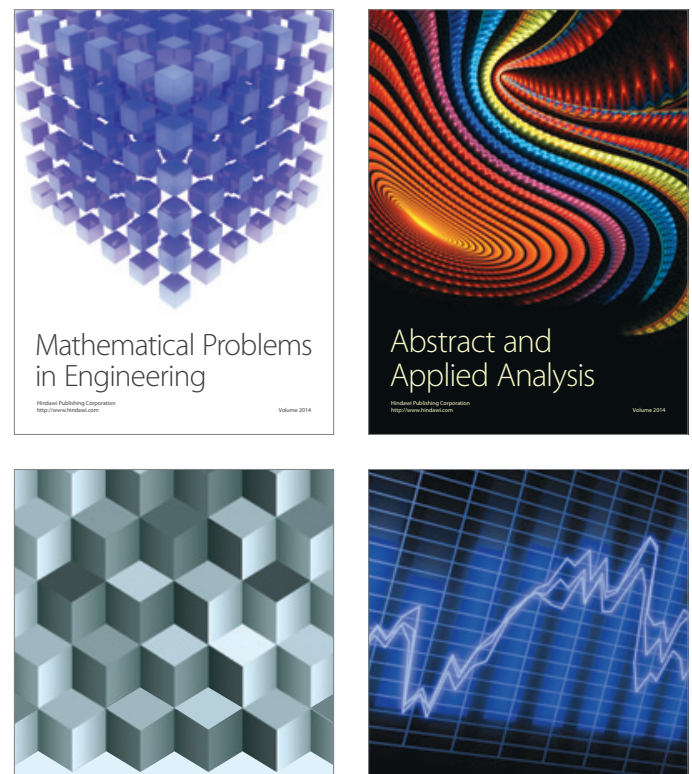

Journal of

Function Spaces

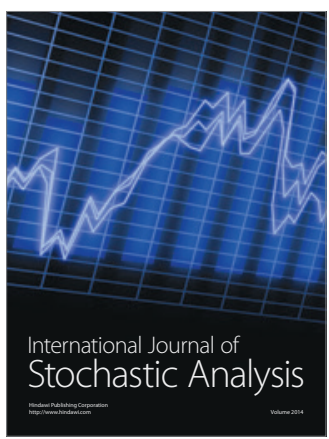

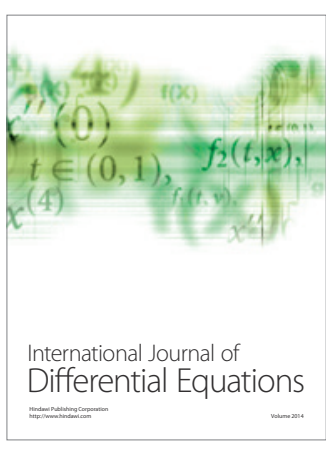
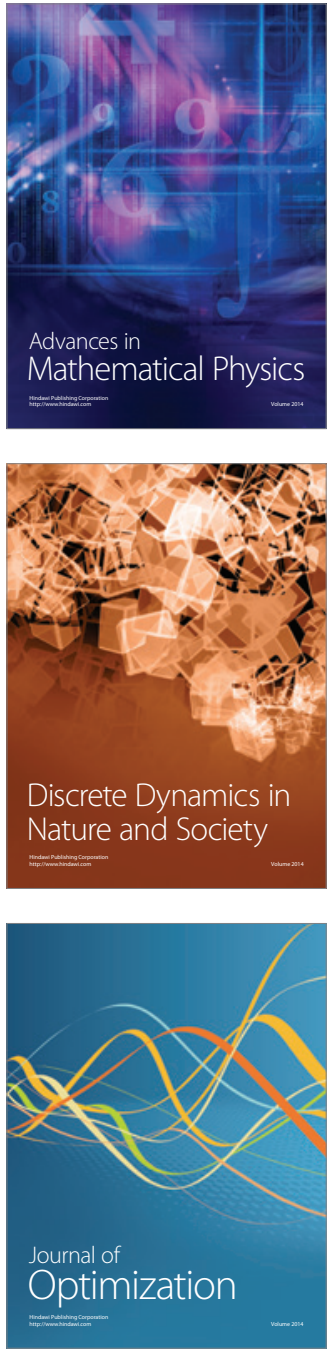